\title{
BRONCOASPIRACIÓN EN EL NIÑO. ROL DIAGNÓSTICO DE LOS ESTUDIOS DE IMÁGENES
}

\author{
Aspiration in children. Role of diagnostic imaging studies \\ María José Barker M. ${ }^{1}$, Dr. Cristián García B. ${ }^{2}$ \\ 1 Interna $7^{\circ}$ Año, Facultad de Medicina Universidad de los Andes. \\ 2 Profesor Titular. Departamento Radiología y División Pediatría, Pontificia Universidad Católica de Chile.
}

\begin{abstract}
ASPIRATION IN CHILDREN. ROLE OF DIAGNOSTIC IMAGING STUDIES
Aspiration is defined as the entrance of foreign material through the respiratory tract to the lungs. This syndrome is relatively frequent in children and often underdiagnosed. It can occur at any age and may cause acute or chronic lung disease. Clinical symptoms depend on the amount and frequency of events, individual response, the kind of aspirated material and the underlying cause. There are different diseases that can cause aspiration. Swallowing disorders, often associated with neuromuscular diseases are a common causal factor. Imaging studies can play an important role in the diagnostic approach and may identify aspiration cause. Among these studies, the most important is videofluoroscopy which allows a dynamic and real-time study of swallowing. Chest radiography and computed tomography can support the diagnosis.
\end{abstract}

Key words: respiratory aspiration, aspiration pneumonia, fluoroscopy, deglutition disorders

\section{RESUMEN}

La broncoaspiración corresponde a la entrada de material extraño a la vía aérea y pulmones. Es un síndrome relativamente frecuente y muchas veces subdiagnosticado. Puede presentarse a cualquier edad y ser factor causal de enfermedad pulmonar aguda 0 crónica . El cuadro clínico dependerá del volumen y frecuencia de los eventos, la respuesta individual, el material aspirado y el factor causal. Las causas de broncoaspiración son variadas. Las alteraciones en los mecanismos de la deglución, muchas veces asociadas a enfermedades neuromusculares son un factor causal frecuente. Los estudios de imágenes juegan un rol importante en la aproximación diagnóstica y pueden orientar a la etiología. Dentro de estos el de mayor relevancia es la videofluoroscopía, que permite un estudio dinámico y en tiempo real de la deglución. La cintigrafía también puede ser de utilidad diagnóstica, al igual que la radiografía de tórax y la tomografía computada. Palabras clave: aspiración respiratoria, neumonía por aspiración, fluoroscopía, deglución

\section{INTRODUCCIÓN}

La broncoaspiración se define como la entrada de material extraño a la vía respiratoria, específicamente bajo las cuerdas vocales (1). Su diagnóstico y manejo son trascendentales, por el alto riesgo de complicaciones graves como neumonía recurrente, insuficiencia respiratoria aguda 0 enfermedad pulmonar crónica. Según las series la aspiración puede ocurrir entre $11-40 \%$ de los niños con trastornos de la deglución (1-3). Esta incidencia puede llegar a $80 \%$ en niños con daño neurológico. En centros de referencia neurológicos, la aspiración a vía aérea constituye el $8 \%$ de las causas de hospitalización por neumonía recurrente (3). La broncoaspiración se manifiesta en un amplio espectro: desde una aspiración masiva, habitualmente de contenido gástrico, la cual causa síntomas agudos, incluida falla respiratoria, hasta el otro extremo de una aspiración crónica, que causa síntomas respiratorios crónicos o recurrentes. Estos

\section{Correspondencia:}

Dr Cristián García B

Departamento Radiología

Pontificia Universidad Católica de Chile

Marcoleta 367 - Santiago

E-mail: cgarcia@med.puc.cl. últimos pueden ser tos prolongada, disnea, hipersecreción bronquial, sibilancias persistentes (3-5).

Es importante destacar la aspiración silenciosa, que corresponde a una aspiración que no gatilla tos ni dificultad respiratoria. Se plantea que este fenómeno podría ser causado por incoordinación de la musculatura faringo-laríngea, con alteración en el reflejo de la tos. Este cuadro corresponde un desafío diagnóstico, ya que al ser asintomática, no se detecta clínicamente y su diagnóstico se basa en la sospecha clínica en pacientes de riesgo: enfermedades neurológicas, musculares, alteraciones de la cavidad oral, la faringe y el esófago, y prematurez $(4,5)$.

\section{TRASTORNO DE LA DEGLUCIÓN}

Son muchas las causas de aspiración en niños, incluyendo los trastornos de la deglución secundarios a trastornos neuromusculares 0 inmadurez de los primeros meses de vida, hendidura laríngea, fístula traqueoesofágica en $\mathrm{H}$, reflujo gastroesofágico (RGE), cuerpo extraño esofágico, acalasia, anillos vasculares, compromiso de conciencia y otras (4). 
La presencia de alguna alteración neuromuscular constituye el principal factor de riesgo de broncoaspiración. El estudio de la deglución es clave para el diagnóstico y el manejo de estos pacientes, para prevenir complicaciones y lograr una alimentación segura(4).

En cuanto al RGE, es una patología frecuente en pediatría y se ha descrito que hasta un $26 \%$ de los pacientes presentan síntomas respiratorios y $2 \%$ crisis de cianosis. Sin embargo se desconoce la relación estadística entre aspiración y RGE (6).

El proceso normal de la deglución se divide en cuatro fases. La preparación oral que corresponde a la primera fase, es voluntaria y es donde se prepara el bolo alimenticio. Se sigue con la fase oral, también voluntaria, donde se produce la elevación de la lengua y propulsión del bolo hacia la faringe. La tercera fase 0 fase faríngea es involuntaria y en ella se eleva el velo del paladar con lo que se oblitera la rinofaringe, se abre el esfínter esofágico superior, se cierra la glotis, hay propulsión lingual y se contrae la faringe. Con esto, se guía el bolo alimenticio desde la boca al esófago, atravesando la faringe y evitando desvíos hacia las fosas nasales o laringe. Finalmente en la fase esofágica de manera involuntaria el bolo avanza por las ondas peristálticas, lo que va seguido de la apertura del esfínter esofágico inferior y posterior pasaje hacia el estómago. Se requiere la coordinación integrada y sincronizada de cada estructura anatómica y de cada una de estas fases para la efectividad y seguridad de los mecanismos de la deglución (7).

Por la complejidad de estos mecanismos y la afectación de distintos sistemas como consecuencia de estas alteraciones, la evaluación, el diagnóstico y el tratamiento de estos pacientes deben llevarse a cabo por un equipo multidisciplinario que incluya el pediatra, gastroenterólogo, otorrino-laringólogo, radiólogo, fonoaudiólogo, nutricionista, psicólogo, y terapista ocupacional (7)

\section{ESTUDIOS DE IMÁGENES}

\section{Estudio de los mecanismos de la deglución mediante Videofluoroscopía}

Este es el método considerado gold standard para evaluar las fases oral, faríngea y esofágica de la deglución $(3,4,8,9)$. Es un método radiológico dinámico, utiliza medio de contraste baritado para la evaluación de la competencia y seguridad de la mecánica de deglución. Se usa fluoroscopía y el proceso es registrado en un formato de video. Los pacientes son estudiados en tiempo real durante la deglución, luego de aportar distintos volúmenes y consistencia de medio de contraste ya sea a través de mamadera, cuchara o jeringa. El contraste puede mezclarse con alimentos como yogurt, papilla y dependiendo del caso, se pueden usar sólo concentraciones espesa y diluida 0 agregar concentraciones semiespesa y sólida. La idea del examen es intentar reproducir lo más fielmente posible la técnica de alimentación habitual del niño en su hogar. De esta manera el examen se puede hacer con el niño en decúbito, en posición sentada o en brazos de su madre $(1,3,4)$. En el examen se registran imágenes en proyección el plano lateral y anteroposterior del paciente (Figura 1).

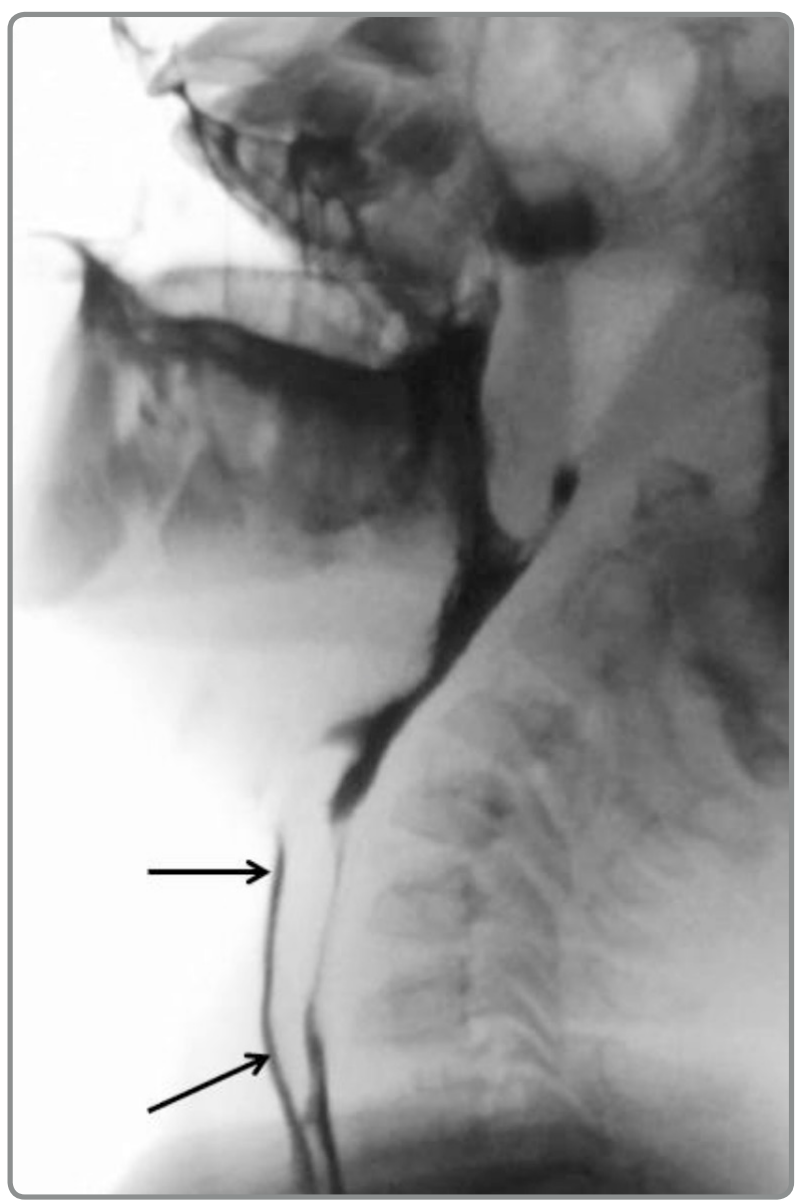

Figura 1.

Niño de 12 años. Sospecha de alteración de la deglución. Estudio de videodeglución muestra pasaje de contraste hacia la tráquea (flechas) durante la administración de contraste diluido

Una de las principales limitaciones de este estudio es la exposición a la radiación, siendo importante su dosificación. Más aún en pacientes pediátricos, con quienes se debe ser más estricto, por la mayor probabilidad que estos presentan de desarrollar consecuencias negativas secundarias a la exposición (8).

Este estudio presenta gran utilidad para decidir la conducta a seguir en relación al manejo de la alimentación del paciente. Decisiones como por ejemplo, suprimir la vía oral en forma temporal o definitiva y alimentar por sonda nasogástrica, o soluciones más drásticas como la alimentación a través de gastrostomía para lograr una nutrición segura, son difíciles en el paciente pediátrico, en parte por el gran impacto emocional que pueden tener en el paciente y su familia $(7,8)$. 


\section{Radiografía de esófago, estómago y duodeno}

Este método utiliza fluoroscopía y consiste también en la administración de medio de contraste baritado por boca, pero es dirigido al estudio anatómico del tubo digestivo alto, al observar el pasaje a través del esófago, estómago y duodeno. Permite detectar estenosis esofágica, hernia hiatal, fístulas traqueoesofágicas (Figura 2), anillos vasculares, malrotación intestinal y evaluar el vaciamiento gástrico. Puede también detectar reflujo gastroesofágico, si bien su sensibilidad y especificidad diagnósticas son bajas en esta patología, en especial en niños menores. También permite evaluar las fases de la deglución, pero su rendimiento es menor comparado con la videofluoroscopía. Su mayor utilidad es aportar información anatómica $(4,7)$ (Figura 2)

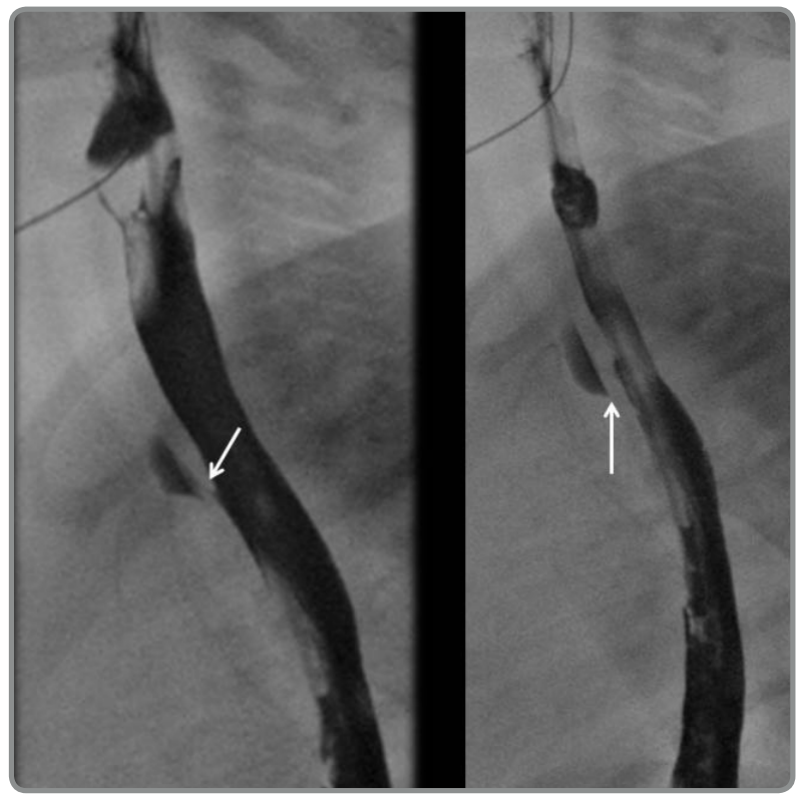

Figura 2.

RN 6 días de vida. Presenta crisis de cianosis con la alimentación. Estudio contrastado de esófago muestra fístula traqueoesofágica "en H", con pasaje de contraste desde el esófago hacia tráquea distal a través de un fino trayecto fistuloso (flechas).

\section{Radiografía (Rx) de tórax}

La Rx de tórax, con proyecciones anteroposterior y lateral es fundamental en el estudio de broncoaspiración en el niño. En el caso de la neumonía por aspiración se puede observar infiltrado difuso alveolar bilateral, con compromiso lobular o segmentario. Si bien su localización puede cambiar según la posición del paciente al momento de la aspiración, característicamente compromete las porciones dependientes del pulmón y es con mayor frecuencia bilateral $(4,7)$ (Figura 3).

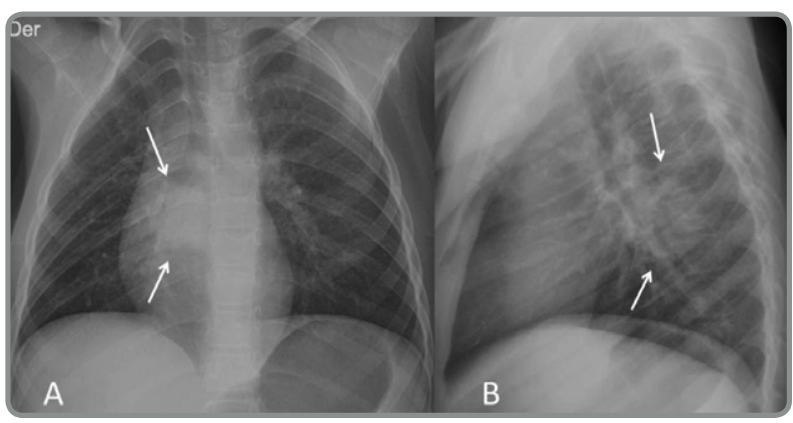

Figura 3.

Niño de 9 años con daño neurológico. Rx de tórax AP (A) y lateral (B) muestran un foco de neumonía aguda del lóbulo inferior derecho (flechas).

Cuando es unilateral, compromete generalmente los lóbulos medio e inferior del pulmón derecho y respeta el pulmón izquierdo, debido a la anatomía del bronquio intermedio derecho $(1,3)$. Puede confundirse con edema pulmonar si bien pueden ser más localizadas. También puede mostrar sombras intersticiales bilaterales de distribución central, hiperinsuflación pulmonar y zonas de atelectasia (4).

El diagnóstico muchas veces es presuntivo y se basa en los antecedentes clínicos, particularmente en niños con factores predisponentes como daño neurológico, retraso mental, síndrome convulsivo, tumores cerebrales, estado post anestésico, excepto en aquellos casos donde es posible presenciar en directo un episodio de aspiración y desarrollo posterior de neumonía.

La aspiración crónica o recurrente es más difícil de confirmar y requiere evaluación cuidadosa de los antecedentes del paciente. Puede manifestarse como un compromiso intersticial bilateral que puede simular otras patologías como asma bronquial o fibrosis quística en etapa precoz (Figura 4).

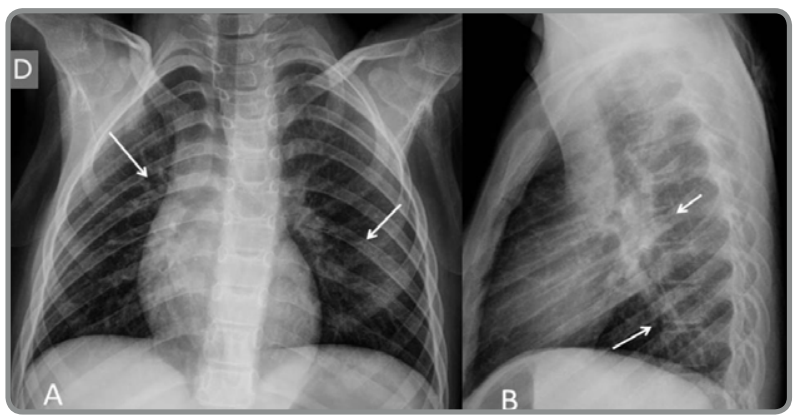

\section{Figura 4.}

Niña de 10 años con daño neurológico e historia de neumonía recurrente. Rx de tórax anteroposterior (A) y lateral (B) muestra sombras intersticiales en ambos pulmones (flechas), sugerentes de aspiración crónica. 


\section{Tomografía Computada de tórax (TC)}

La TC de alta resolución es de utilidad en el diagnóstico diferencial y en especial en la documentación del daño del parénquima pulmonar una vez hecho el diagnóstico. Ese examen utiliza radiación ionizante, por lo que el examen se debe hacer con dosis lo más bajas posibles y monitoreado por un médico radiólogo. Es un examen rápido y puede requerir sedación según la edad. Puede mostrar áreas focales o múltiples de compromiso alveolar, bronquiectasias, opacidades "en árbol en brote", compromiso intersticial, con mayor frecuencia en los lóbulos inferiores (4) (Figura 5).

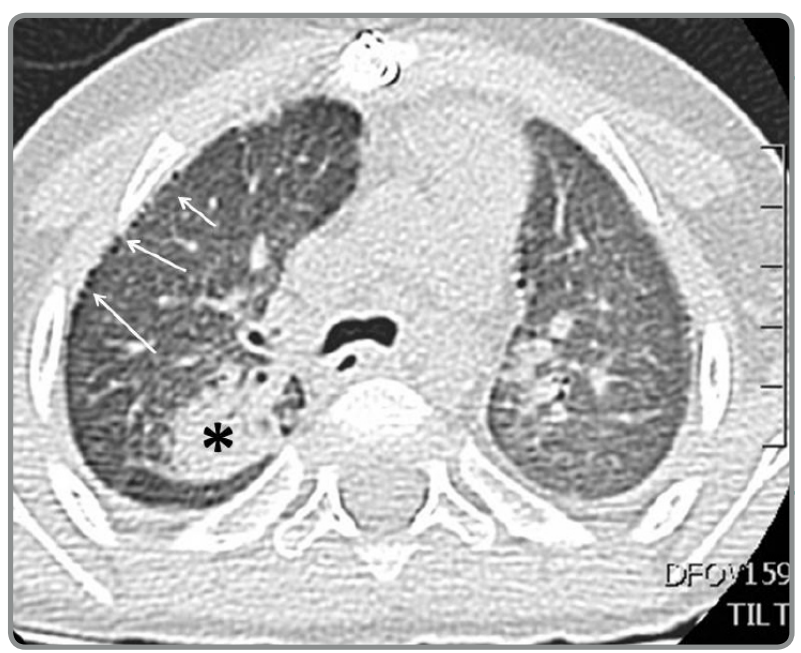

Figura 5.

Lactante de 17 meses de edad con síndrome de Down, cardiopatía congénita operada, aspiración a vía aérea confirmada con estudio videodeglución previo. Tomografía computada de tórax con corte axial a nivel de la carina, muestra zona de neumonía aguda en el segmento posterior del lóbulo superior derecho $\left({ }^{*}\right)$. Hay además opacidades intersticiales bilaterales y numerosos pequeños quistes subpleurales (flechas)

\section{Cintigrafía para detección de aspiración}

La cintigrafía es un método no invasivo, de baja radiación y bien tolerado por el paciente, aunque no se utiliza de rutina. Puede ser utilizado para la detección de aspiración pulmonar, con mayor frecuencia en niños con antecedente de RGE. Puede usarse esencialmente como complemento de un estudio contrastado de esófago, estómago y duodeno para descartar una lesión anatómica y una videofluoroscopía para evaluar la deglución (3). Tiene baja sensibilidad para la detección de microaspiración.

\section{Resonancia magnética (RM)}

La RM es especialmente útil en el diagnóstico de condiciones que afectan secundariamente la deglución, como alteraciones del sistema nervioso central con compromiso de los pares craneanos y la motilidad esofágica. Es decir, ayuda en la correlación del trastorno neurológico con la anatomía y la función de la deglución (5).

También puede ser de utilidad en el estudio de lesiones cervicales que pueden comprometer la dinámica de la deglución. Este examen no utiliza radiación, pero es un examen relativamente largo y en la mayoría de los casos puede requerir sedación. No tiene buen rendimiento en la evaluación del parénquima pulmonar.

\section{CONCLUSIÓN}

El síndrome de broncoaspiración es frecuente y muchas veces sub-diagnosticado en el niño, llevando a consecuencias fatales si no es tratado a tiempo. Es importante el diagnóstico precoz para ofrecer un tratamiento adecuado, previo generar daño pulmonar irreversible.

Los métodos de imágenes son de gran utilidad para el diagnóstico y manejo de sus complicaciones en especial el estudio de videodeglución y la Rx de tórax. Permiten además una orientación pronóstica, claves al momento de decidir el método de alimentación más segura de estos niños y evaluar la necesidad de suprimir alimentación por vía oral de manera transitoria o incluso permanente, que son situaciones de gran impacto para el niño y su familia.

Es importante mantener una constante revisión en estos métodos de estudio, para así poder ofrecer la manera más segura y eficaz de diagnóstico para estos pacientes.

Los autores declaran no tener conflicto de intereses

\section{REFERENCIAS}

1. Weir K, McMahon S, Barry L, Ware R, Masters IB, Chang A. Oropharyngeal aspiration and pneumonia $\mathrm{n}$ children. Pediatr Pulmonol 2007;42:1024-1031

2. Bravo $P$, Olate $M$, Vega-Briceño $L$, Muñoz $B$, Holmgrem $L$, Sánchez I. Características clínicas, epidemiológicas, y factores asociados al diagnóstico de neumonía recurrente en niños, experiencia de12 años. Rev ChilPediatr 2004: 75: 434-40

3. Owayed AF, Campbell DM, Wang EEL. Underlying causes of recurrent pneumonia in children. Arch Pediatr Adolesc Med 2000;154:190-1943

4. de Benedictis FM, Carnielli VP, de Benedictis D. Aspiration lung disease. Pediatr Clin N Am $2009 ; 56: 173-190$

5. Sheikh S, Allen E, Shell R, Hruschak J, Iram D, Castile R, McCoy K. Chronic aspiration without gastroesophageal reflux as a cause of chronic respiratory symptoms in neurologically normal infants. Chest 2001;120:1190-1195

6. Rossel M, Ceresa S, Orellana P, Pino C, González P, Olea $E$, Araya $M$, Espinoza J. Cintigrafía esofágica y pulmonar en niños con reflujo gastroesofagico y enfermedad pulmonar. Rev ChilPediatr 1985;56:35-387.

7. Henao PA, Lopera MV, Salazar OF, Medina PA, Morales OL. Guía de práctica clínica basada en la evidencia para el diagnóstico de disfagia en niños. IATREIA 2009; 22:169-179

8. Gates J, Hartnell G, Gramigna D. Videofluoroscopy and Swallowing Studies for

Neurologic Disease: A Primer. RadioGraphics, 2006; 26: e22

9. DeMatteo C, Matovich D, Hjartason A. Comparison of clinical and videofluoroscopic evaluation of children with feeding and swallowing difficulties. Dev Med Child Neurol 2005;47:149-157 